\title{
RESISTOR GEOMETRY COMPARISON WITH RESPECT TO CURRENT NOISE AND TRIM SENSITIVITY
}

\author{
W. ULBRICH \\ Lehrstuhl für Netzwerktheorie und Schaltungstechnik, Technische Universität München, München, \\ W. Germany $\dagger$
}

(Received March 30, 1977)

\begin{abstract}
The paper deals with laser trimming of film resistors. The finite-difference solution of the electric field calculated by a digital computer gives the total resistance and the inhomogeneous current distribution within the resistor area. The current noise voltage was found to be proportional to the sum of reciprocal subareas wherein the current density is approximately constant. Resistor trimming is modelled by finite increments of cut length to obtain the resistance trim rate and the trim sensitivity.

Known and new resistor geometries and cut configurations are compared (based on the same resistor area and the same initial tolerance of $\pm 20 \%$ ) to find the optimal geometry depending on the ratio of the nominal resistance value to the sheet resistivity. Diagrams are given leading to practical design rules not only for resistance trimming but also for deterministic and functional tuning.
\end{abstract}

\section{SIMULATION TECHNIQUE}

The approach used for the computer simulation is based on the over-relaxation method for solving finite-difference equations. It results in an excellent approximation to the Laplacian differential equation for the electric potential $\Phi$ within the distributed

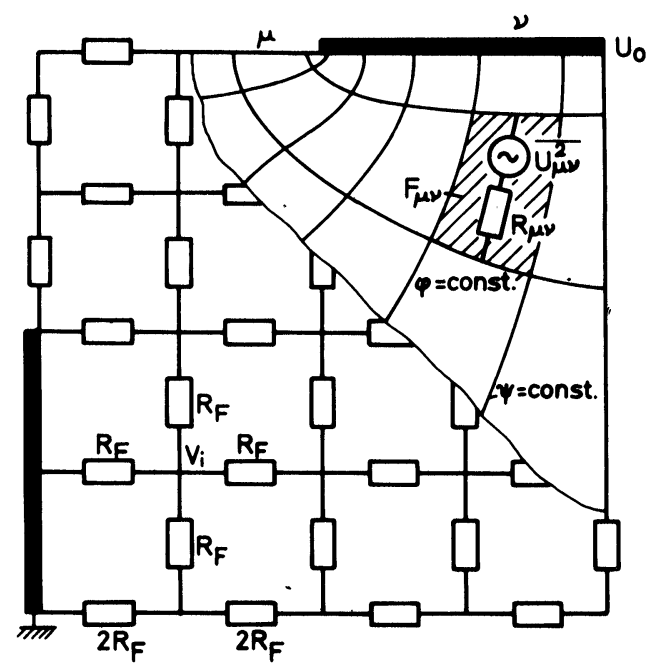

FIGURE 1 Film resistor model.

$\dagger$ Arcisstrasse 21, 8 München 2. resistive film. For the first simple model, a constant sheet resistivity $R_{F}$ of a two dimensional, piecewise rectangular bordered region is assumed. The boundary conditions are either ideal conductivity $(\sigma=\infty)$ at terminations or zero conductivity $(\sigma=0)$ at the other borders including the edges of trim cuts with a narrow kerf. 'The finite-difference solution is similar to that of Walter ${ }^{1}$ and can be understood with the aid of an equivalent equidistant lattice network of lumped resistors $R_{F}$ as shown on the left of Figure 1. Starting with a fixed potential difference $U_{0}$ between the terminations, the program computes all node voltages $V_{i}$ which represent selected potentials $\Phi_{i}$ at corresponding physical points of the region. A result is the total resistance $R=U_{0} / I$ obtained by determining the total current flow $I$ across a suitable crosssection of the equivalent lumped network. In determining other properties such as current noise, power dissipation and nonlinearities, the inhomogeneous current distribution within the resistor area becomes important. A two-dimensional interpolation between known discrete potentials $\Phi_{i}$ yields a set of $n$ equidistant potential lines $\Phi=$ const. Furthermore, an orthogonal set of $n$ equidistant current flow lines $\psi=$ const. is constructed. Both sets of lines together dissect the resistor area in $n^{2}$ subresistors with the same resistance $R_{\mu \nu}=R(=2 R, R / 2$ at the borders) as shown in Figure 2, for example. Such a plot is a good qualitative indication for hotspots or unused subareas. For quantitative results, the current noise is chosen. 


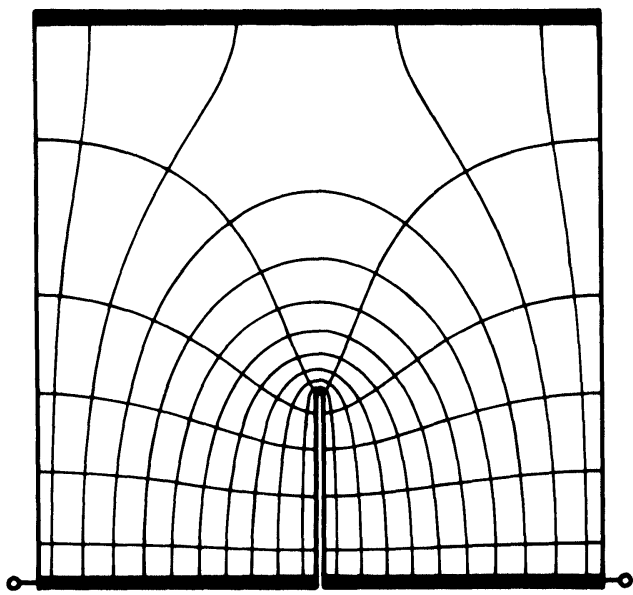

FIGURE 2 Potential and current flow lines.

The current noise voltage $\overline{U^{2}}$ of a resistor has been shown by Kuo and Blank ${ }^{2}$ to be strongly dependent on geometry: $\overline{U^{2}}=U_{0}^{2} k / F_{e}$ where $k$ is a material constant and $F_{e}$ is the effective area of the film resistor. To determine $F_{e}$ it is practical to consider $n^{2}$ subresistors $R_{\mu \nu}$ with individual subareas $F_{\mu \nu}$ as noise sources $U^{2} \sim 1 / F_{\mu \nu}$ as shown on the right of Figure 1. Combining them in series and parallel it follows that:

$$
\frac{1}{F_{e}}=\frac{1}{n^{4}} \sum_{\nu=1}^{n} \sum_{\mu=1}^{n} \frac{1}{F_{\mu \nu}}
$$

According to the Quan-Tech standard the current noise index $A=10 \mathrm{lg} \overline{U^{2}} / U_{0}^{2}$ is computed with reference to the current noise index $A_{0}$ of a norm area $F_{0}$ with a homogeneous current distribution $\Delta A=A-A_{0}$.

\section{TRIM CHARACTERISTICS, TRIM SENSITIVITY, NOISE INDEX VARIATION}

The computer program, written for usual and new resistor geometries, was applied to a series of different cut lengths $l$ to model the trim process. The trim characteristic is the plot of the normalized resistance $R / R_{F}$ (or $R / R_{B}$ ) as a function of the normalized cut length $\lambda=l / L$ where $L$ is the maximal length. Usually, one diagram involves several functions for one principal resistor form but different aspect ratios. An ideal characteristic shows a small uniform gradient in a greatest possible range of resistance values. This gradient, designated as trim sensitivity

$$
S=\frac{d R / R_{F}}{d l / L},
$$

is a good measure for the resistance precision obtained when switching off the laser beam. The variation of the current noise is also plotted as a function of the normalized cut length $\lambda$. Such a function can have a maximum or a minimum or both.

Figures 3 and 5 show trim characteristics of the $U$ form without or with a conductive roof. New geometries, the angle and $3 S C$ (on $\underline{3}$ sides $\underline{c}$ onducted), arise by halving the $U$-form and cutting around the conductive borders. Figures 4 and 6 show the corresponding noise diagrams. Other geometries are also examined.

\section{RESISTOR GEOMETRY COMPARISON}

According to a qualitative comparison, the usual tophat form always appears more disadvantageous than the $U$-form. Next, six different geometries (bar resistor with single cut or $L$-cut, $U$ - or angle-forms etc.) with the same resistor layout area $F_{0}=16 \mathrm{~mm}^{2}$, the same initial tolerance of $\pm 20 \%$ and the same trim conditions (trim speed $10 \mathrm{~mm} / \mathrm{s}$, stop time $1 \mathrm{~ms}$ ) are compared. The turning point of the $L$-cut is chosen to be theoretically optimal with a maximal effective area $F_{e}$ after trimming.

Figures 7 and 8 show the obtainable tolerances and the current noise index as a function of the ratio of the final resistance $R_{f i n}$ to sheet resistivity $R_{F}$, presuming the optimal design given in Figure 9. Curves for maximum, medium, and minimum values are plotted for each form. The optimal geometry depends on the ratio $R_{f i n} / R_{F}$. In the ranges $R_{f i n} / R_{F}$ $<0.5, R_{f i n} / R_{F}>6$ the single straight-in cut $l$ (the $U$-form 5 , usefully with a pre-printed kerf) is adequate. If the ratio $R_{\text {fin }} / R_{F}$ is between 1 and 5 , no ideal geometry exists. Therefore, additional aspects must be considered (computer facility for the trimming system, stability, conductive terminations). Multiple straight-in and $L$-cuts require efficient prediction algorithms to control the trim path so that the desired resistance value is reached while the sensitivity is low. New forms 2, 3 and are better suited for trimming with smaller computer facilities. Problems may arise with cuts too near to the terminations of thickfilm resistors. 

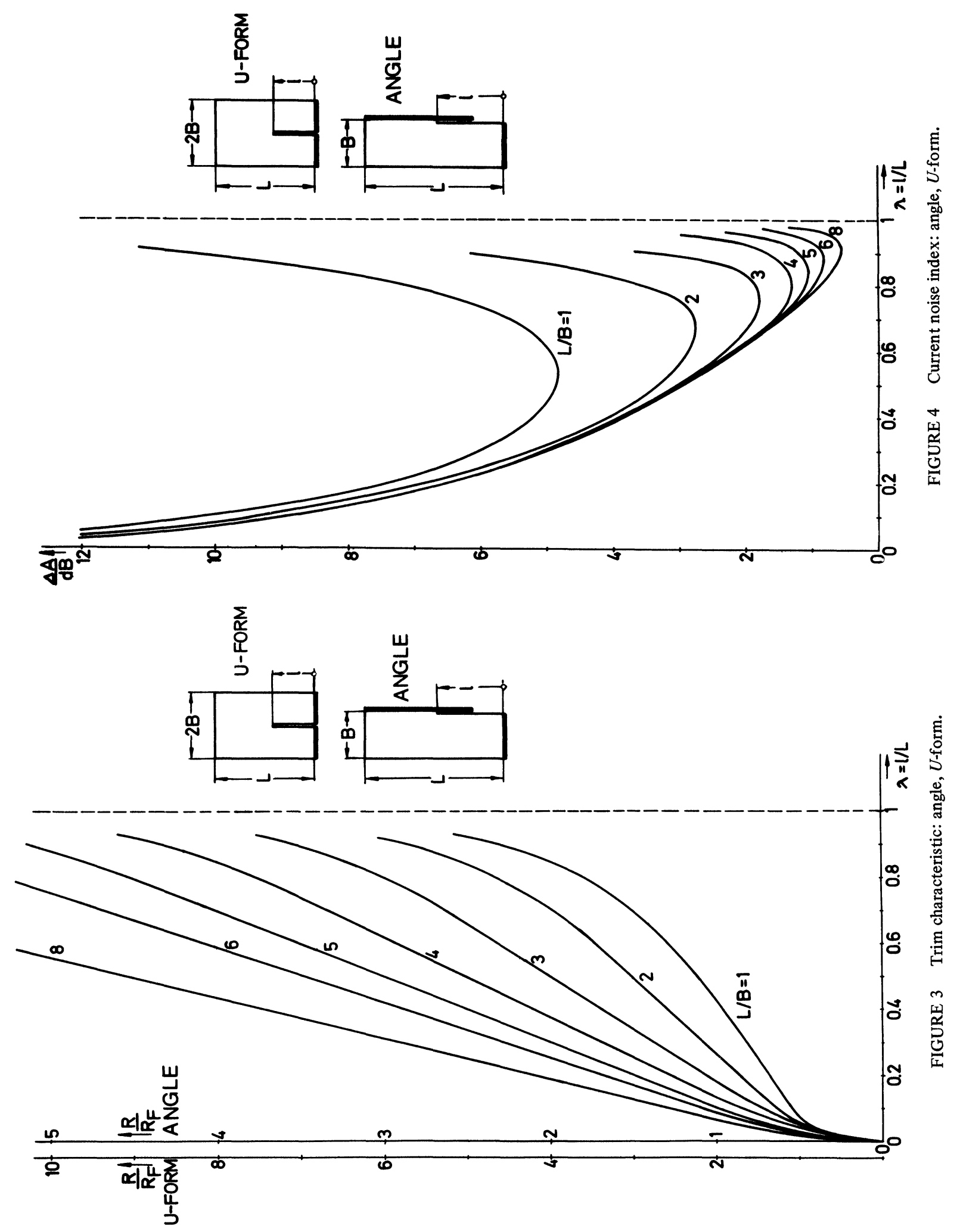


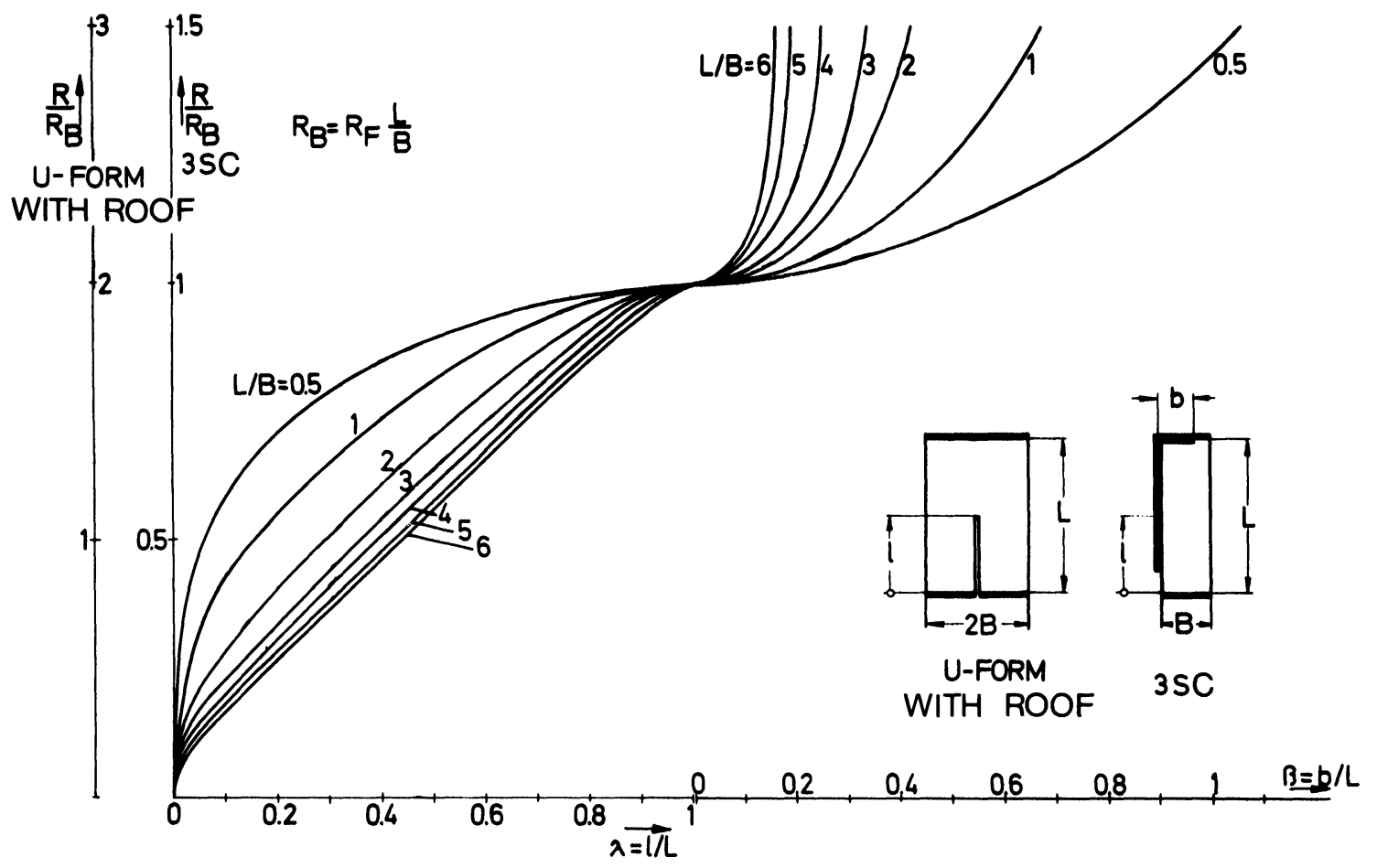

FIGURE 5 Trim characteristic: $U$-form with roof, $3 S C$.

$\left.\frac{\Delta A}{d B}\right\}$

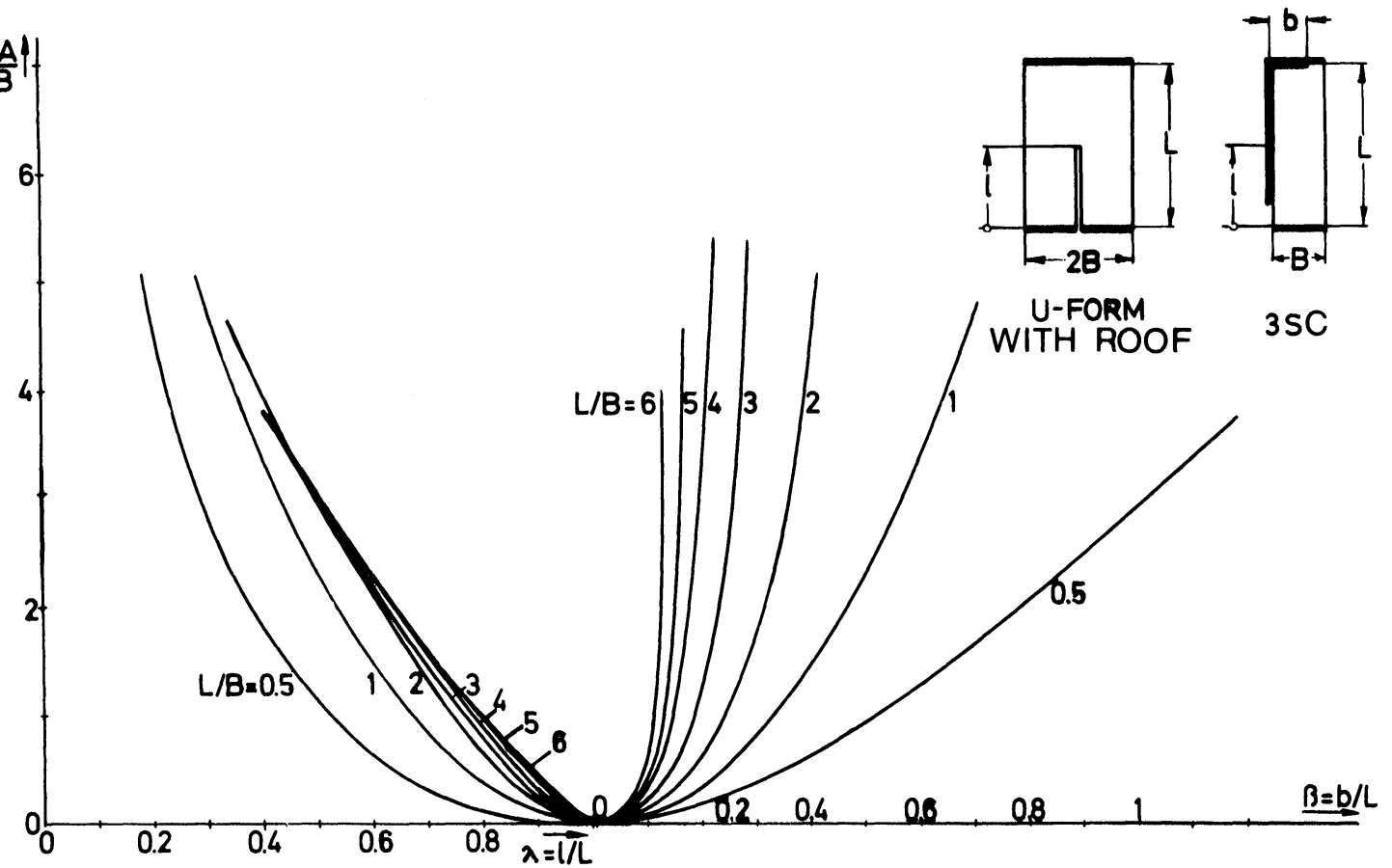

FIGURE 6 Current noise index: $U$-form with roof, $3 S C$. 

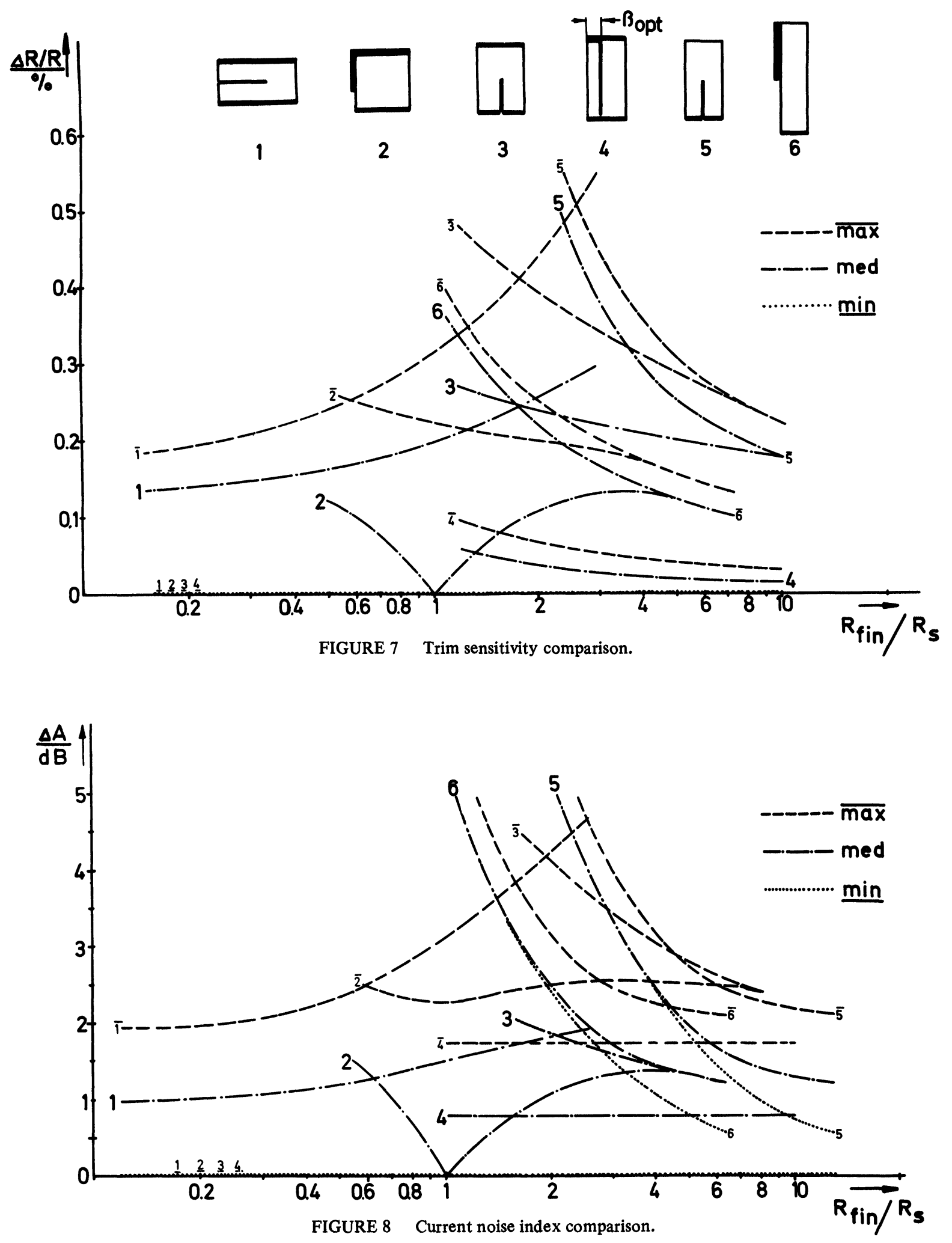


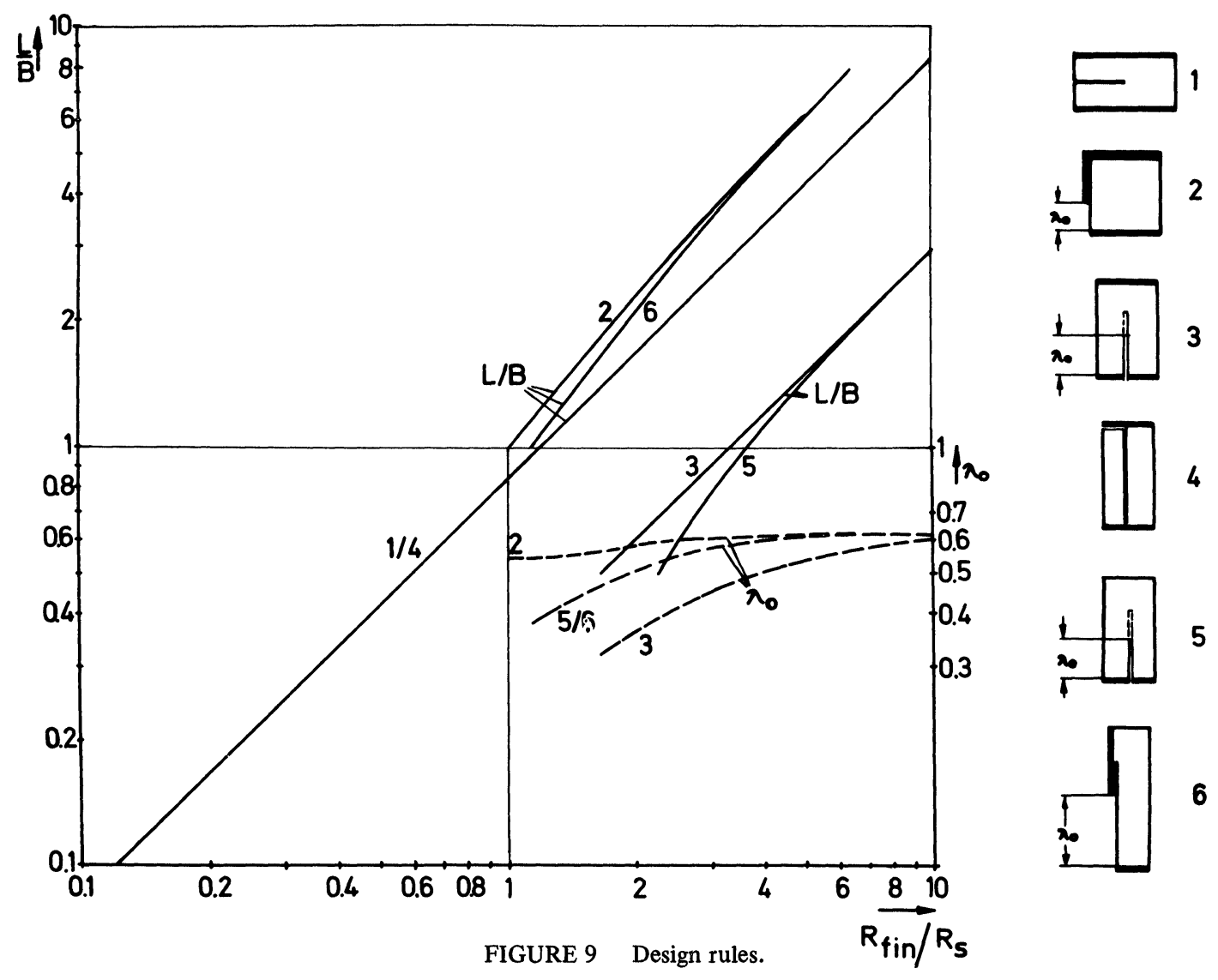

\section{CONCLUDING REMARKS}

For functional tuning at selected resistors additional aspects are to be taken into account. The final resistances $R_{f i n}$ are usually unknown. Hence, we prefer simple trim configurations 1,3 and 5 with a large resistance trim rate. Taking into account the greater stop times of functional tuning bridges, not only a small, but rather a constant trim sensitivity is desirable to choose a constant predicted stop limit.

Using computer simulation, numerous variations of geometries and undisturbed noise determinations are possible. Note that the computed variations of current noise depend only on the variations of the current distribution and are not affected by an increase in noise caused by rough trim cut edges. Test results show a very good agreement between computation and measurement.

\section{REFERENCES}

1. D. W. Walter, Computer simulation of resistor trimming, 23rd Electr. Components Conf., pp. 56-62 (1973).

2. C. Y. Kuo, H. G. Blank, The effects of resistor geometry on current noise in thick-film resistors, Symp. on Hybrid Microelectronics, pp. 153-160 (1968). 

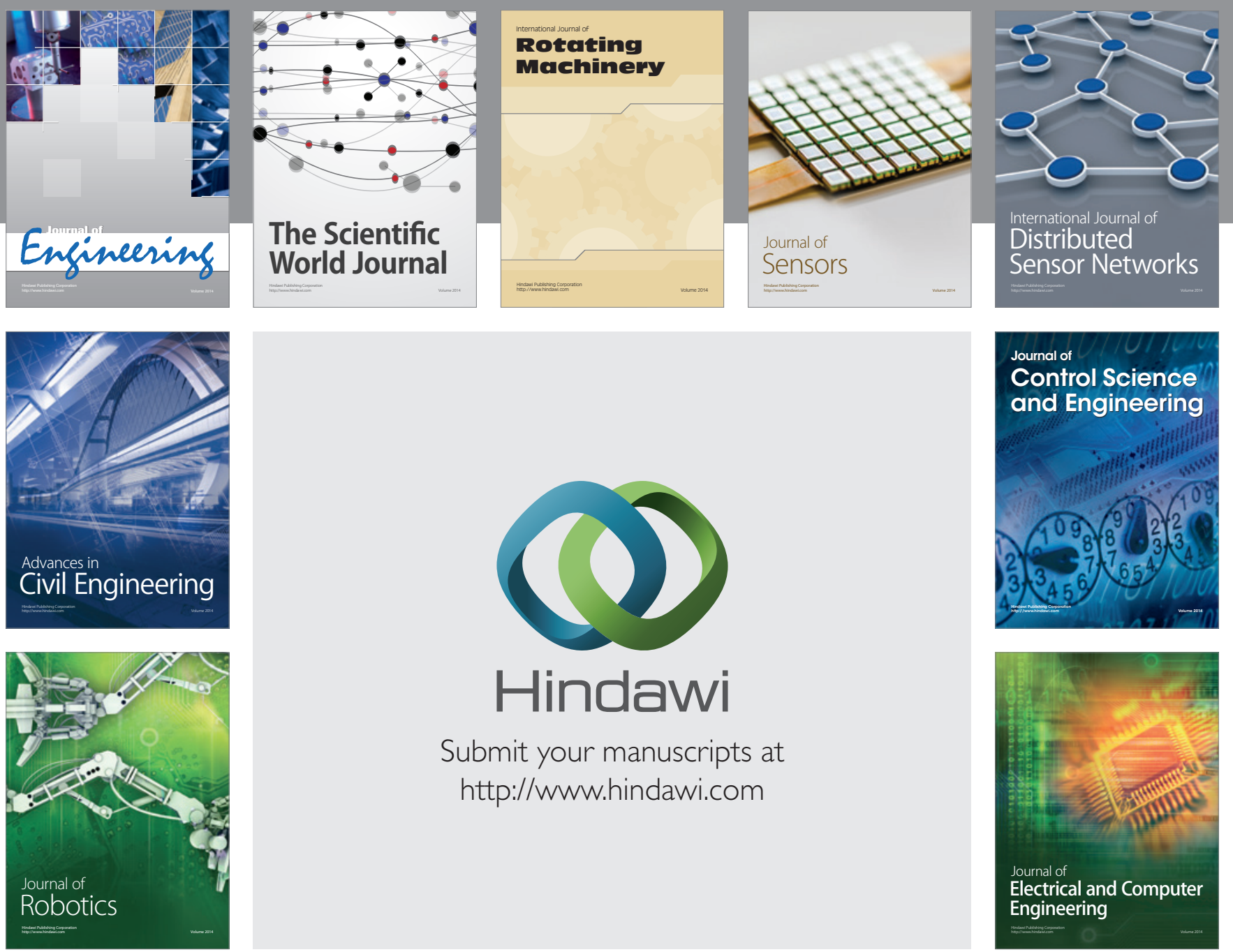

Submit your manuscripts at

http://www.hindawi.com
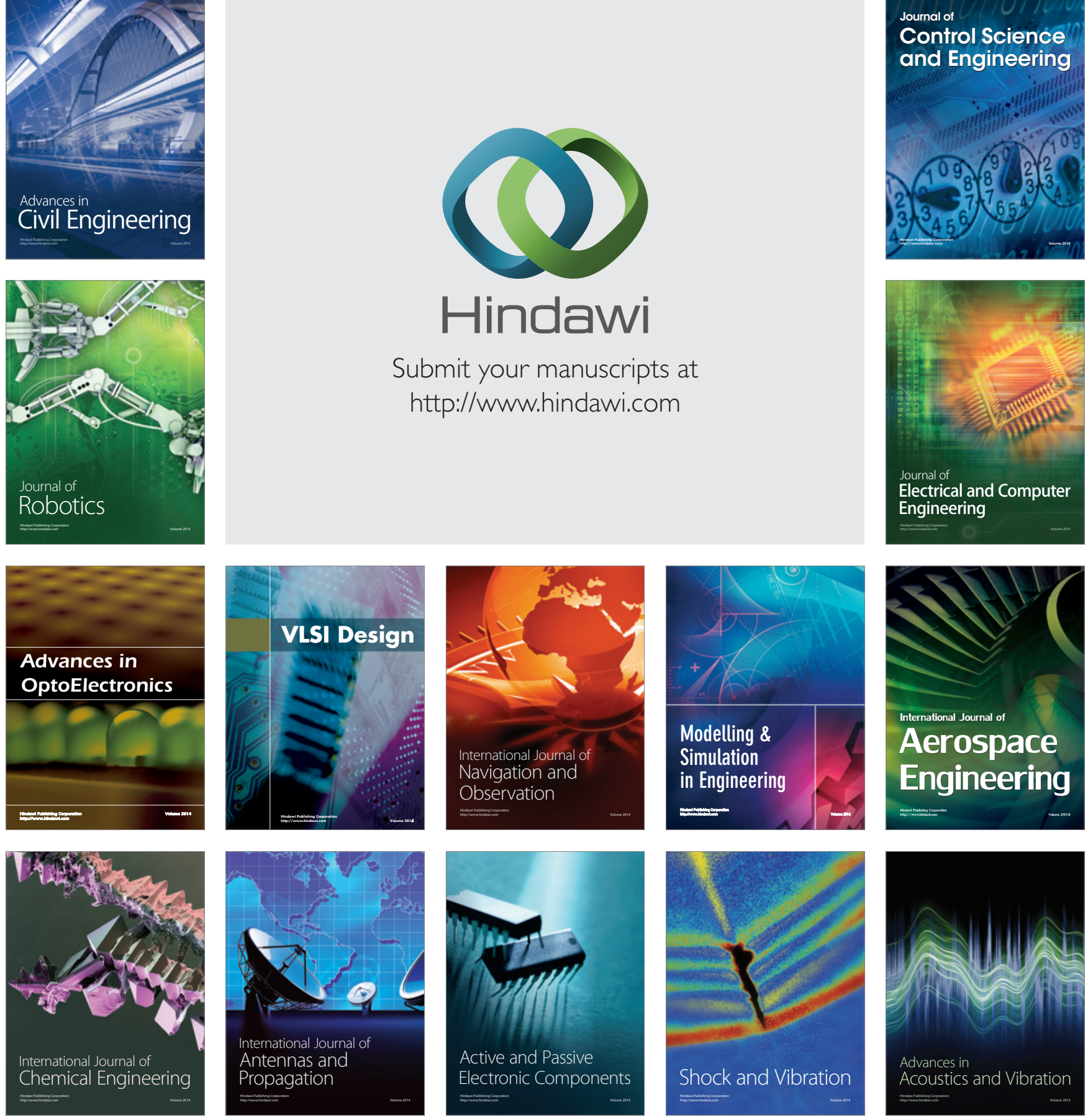